\title{
WAYS TO STANDARDIZATION \\ IN ELECTROPHORESIS ARE BROUGHT TO LIGHT
}

A workshop entitled Electrophoresis Standardization: Approaches and Needs, drew 54 participants to the National Bureau of Standards (NBS) June 25,1984. Co-sponsored by NBS and the Electrophoresis Society of the Americas, the meeting was hosted by the NBS Center for Analytical Chemistry.

The participants, many of whom came in con. junction with an International Symposium on Electrophoresis in Quantico, VA, organized by the Federal Bureau of Investigation, represented leadership in various scientific sectors. They included 15 representatives from industry and 8 from universities with the remainder from government agencies including State crime laboratories, the National Institutes of Health, the Uniformed Services University of the Health Sciences, and the Food and Drug Administration.

The first series of talks discussed various needs for standardization. Subsequent talks described how different laboratories approach standardization. Summaries of some of the talks are given in this review. A monograph containing edited versions of the papers was scheduled for release this spring.

Dr. Robert Allen, Medical University of South Carolina, gave an introductory talk that addressed some of the current directions in electrophoresis. The recent mandates by many states to perform electrophoresis on the blood of newborns will have a dramatic impact on the need for interlaboratory comparisons. This suggests also that there will be needs for standardized materials, standard protein mixtures, and specifications for materials purity.

Dr. Berthold Radola, visiting from the Technische Universität München, Federal Republic of Germany, discussed an important new concept for the standardization of thin layer isoelectric focusing (TL-IEF). He described a new technique for bonding and drying acrylamide on a polymer film. One variable in TL-IEF techniques is the unpredictable starting condition of voltage and current flow because of varying amounts and types of impurities present in the casting mixtures due to different acrylamide sources.

Preparation of acrylamide gels involves polymerization of acrylamide and results in variable amounts of persulfate in the gel. The process of bonding and drying acrylamide for producing rehydratable gels includes a series of washes that removes unpolymerized material, impurities, and excess persulfate from the casting mixture. The resulting TL-IEF medium is rehydrated in the appropriate ampholyte mixture to produce gels that give reproducible results. The initial voltages are greater and focusing times can be shortened because of higher field strengths. The availability of these materials from a commercial source should allow many laboratories to be able to perform TL-IEF analyses using comparable gel substrates.

Dr. Bruce Budowle from the Federal Bureau of Investigation described a modified procedure for determining phenotypes of phosphoglucomutase. This enzyme is important in the forensic field because of the possibility of discriminating certain individuals from others on the basis of blood stains found at the scene of a crime of violence. The initial procedure developed was reproducible based on results from over 100 runs. However, changes occurred in the banding patterns after the deionizing cartridge in the purified water system was changed. It appears that contaminants in the original system were helping to achieve the original, reproducible separations. With a highly purified water (10-14 megohm resistivity), reproducibility was lost. This is particularly disturbing because the original results could not be reproduced by adding back known impurities such as sodium chloride. However, it was generally suggested that reproducibility depended upon the capability of having a known, controlled system and that defining electrophoretic solutions based upon the purest water was the best approach.

Dr. Carl Merril of the National Institutes of Health described the historical aspects of the development of the silver stain for electrophoretic gels. He also gave evidence for a number of mechanisms by which silver stain works. He suggested that the standardization of a silver stain was going to be difficult because of the variety and combination of stains and electrophoretic separations used in laboratories today and the reluctance of laboratories to switch from a system they are accustomed to using. However, he sug. 
gested that one of the more promising ways to achieve standardization is the use of a normalization procedure for doing quantitative work and the development and use of standardized protein methods for absolute quantitation.

One of the difficulties in quantitation is making sure that the stained protein spots are not saturated with silver. A result of saturation is that the tops of the densitometric tracings are "clipped"; consequently, there is no Gaussian distribution of stain in the spot. Linear results can be achieved if precautions are taken to make sure that the proteins are not saturated. Each protein has a different linear calibration curve which means that the relationship of each protein to a standard has to be known. Thus, in comparing spots on two dimensional gels or bands on one-dimensional gels, it is necessary to compare the same respective protein spots or bands on different gels. One way to overcome variations in quantitation between gels is to choose a set of spots on twodimensional gels that have the same density ratios from one gel to the next. These spots are called operationally consistent. The characteristic values of these spots are then taken to develop a correction factor so that all spots on a gel can be normalized against that set of spots. Individual variations between spots can then be detected and protein changes determined.

The concentration of individual proteins can change for a number of reasons. For example, some human proteins change in a subtle manner as people age. Three known proteins that increase with age are actin, apo-a1-lipoprotein, and haptoglobin. Haptoglobin con-

... the pattern in the gel could be used in much the same way as a photographer uses a step wedge.

centration increases approximately $43 \%$ per decade of life. Some proteins are observed to change when a person contracts Parkinson's disease while others may be expressed as the result of an individual contracting multiple sclerosis.

Dr. Merril suggested that a useful standard could be obtained by carbamylating proteins to produce electrophoretically separated spots of a fixed pattern in an electrophoretic gel. By increasing the concentration of each protein, the pattern in the gel could be used in much the same way as a photographer uses a step wedge. The difficulty with this process is that one would have to make sure that all proteins remained in the gel. One way to correct for such variations is to label radioactively the proteins to provide a second technique to check for protein penetration into the gels. By making appropriate corrections, relative concentrations could be replaced with absolute concentration.

Richard Cook the FMC Corporation discussed applications of agarose in gel diffusion, electrophoresis, chromatography, and cell culture and the requirements for an agarose for use in each of those applications. The requirements for an agarose can be defined in terms of measurable parameters, including clarity, electroendoosmosis (correlated with the amount of ester sulfate), gel strength, various polyanions and their associated hydrated cations, pyruvate, moisture, and gelling temperature. Because many laboratories have specialized needs for certain properties of agarose, it isimpractical to narrow the choices to a single type of agarose. However, with the ability to furnish analytical data for each lot of material, along with an assurance that lot-to-lot variations will be minimal, the industry could provide the researcher with valuable information with which intelligent choices can be made.

Dr. Norman Anderson, Argonne National Laboratories, discussed aspects of standardization that affect

... tighter manufacturing standards would help to avoid currently observed differences.

two-dimensional electrophoresis. He presented results showing standardization of the first dimension separation by isoelectric focusing through the use of carbamylated creatine phosphokinase. These carbamylated proteins differ from one another by a single charge. Use of these proteins yields information about the ampholytes incorporated in the gels to create the $\mathrm{pH}$ gradient. Dr. Anderson showed that different manufacturers' lots of ampholytes produced variable spacing of the separated proteins throughout the isoelectric focusing gel. He selected and procured a large batch of ampholytes that showed the most even spacing in the gel. While this is one way to achieve reproducible run-to-run results, tighter manufacturing standards would help to avoid currently observed differences. In addition, standards ( $\mathrm{pI}$ markers) are still needed to extend the range of proteins into the basic and acidic ends of the isoelectric focusing gel. Currently available pI markers are still limited in their $\mathrm{pH}$ range.

For molecular weight standardization, Dr. Anderson's laboratory uses a homogeneous preparation of rabbit psoas muscle, mainly because many of the rabbit proteins have been sequenced and molecular properties have been described.

Resolution of a two-dimensional gel is more difficult to measure. One approach is to take an average of all the spots on the gel and determine how many 
average-sized spots can be packed into the gel and still maintain resolution. This theoretical measurement gives an indication of how different modifications to the system will improve or degrade performance.

Dr. Anderson then discussed problems in matching distorted gels and in the presentation of data. Pattern recognition is an emerging technology for twodimensional electrophoresis, with the emphasis being placed on identification of individual protein spots.

Dr. George Bers, Bio-Rad Inc., presented information from a thorough literature review of the detergent sodium dodecyl sulfate (SDS) and its effects in electrophoresis. He classified the various potential contaminants of SDS and showed examples of how electrophoresis is affected. He also pointed out the several types of lipophilic contaminants such as the lower alkyl alcohols, alkene hydrocarbons, and the di-alkyl ethers. There are other potential contaminants such as the dialkyl sulfates and alkyl acids that are generally insoluble and tend to show their presence in terms of a cloudy SDS preparation. Other contaminants mentioned were inorganic salts, metals, amino acid contaminants fromfibers, and inorganic acids and bases.

The fact that there is considerable variability in commercial sources of SDS demonstrates the need for strict quality control. Specifications for SDS purity were suggested and currently available materials were identified that match those specifications.

Problems that surface as a result of variable SDS preparations include variations in molecular weight determinations, changes in banding patterns, variable staining of bands, differences in background staining, diffusion of minor bands, and in some cases, the appearance of protein bands that shifted position and were not expected to be seen in that particular position. Other problems attributed to SDS variability were enzyme

. . a process of "detergent engineering" should be evaluated.

renaturation difficulties and inhibition because of lipophilic material being present in the SDS preparation.

Dr. Bers mentioned that autoclaving SDS was a common practice by nucleic acid chemists. However, SDS is hydrolyzed considerably by this process, rendering it useless for protein work.

Where two-dimensional electrophoretic analysis or molecular weight determinations are part of an experiment, it is most likely that a highly purified and highly reproducible SDS preparation will be required. In cases where better resolution is required, and it is known that contaminants enhance the resolution, a pro- cess of "detergent engineering" should be evaluated. That is, known "contaminants" need to be added in known amounts to an SDS preparation to achieve the proper results.

Dr. Steven Spotts, LKB Corp., discussed possible approaches to the standardization of isoelectric focusing using ampholytes. Companies that manufacture ampholytes try to ensure reproducibility by producing large batches and to minimize variations by carefully controlling the synthesis and subsequent fractionation thereof. Companies also distribute application notes or protocols for their particular ampholytes in an attempt

The end goal is to reproduce identical $\mathrm{pH}$ gradients from one run to another.

to optimize the separations process. Attention is given to electrolyte solutions, gel thicknesses, and recommended concentration of the ampholytes. The end goal is to reproduce identical $\mathrm{pH}$ gradients from one run to another. Problems that can arise from inhomogeneous samples, impurities in additives such as urea or other detergents, or impurities in the sample itself were also discussed.

Commercially available pI marker proteins seem to be useful for calibrating pI gradients, but they must be used while fresh. Otherwise, artifacts may arise as the proteins age. Attendees were warned against calibrating pI gradients by direct measurements because of the variabilities resulting from temperature differences. Calibrating the electrodes at $25^{\circ} \mathrm{C}$, and measuring at $4{ }^{\circ} \mathrm{C}$ can cause errors of up to one-half of a $\mathrm{pH}$ unit.

Immobilized $\mathrm{pH}$ gradients recently have been introduced as commercial products to provide powerful methods for protein separation. Because of sampledependent properties that need to be defined, immobilized $\mathrm{pH}$ gradients are not ready to be considered as viable standards for electrofocusing.

Mr. William Gorman, EC Apparatus Co., detailed the current status of the industry's attempts to provide standards for electrophoresis power supplies. He considered a number of variables that could be addressed. Among those that appeared to be the most important were: 1) type of output: DC, pulsed DC with programmed decay, pulsed half-wave or full-wave rectified, or pulsed with filtering; 2) safety features; 3 ) types of readout; 4) output connections; 5) temperature operating ranges; 6) power style: line frequency type or switched frequency; 7) end point detection; 8) degree of regulation; 9) set point controls; 10) testing parameters; 11) serviceability; and, 12) electrical code compliance.

Mr. Gorman's talk provided an important list of 
points for manufacturers to consider as newer devices are built that take advantage of increased voltages in electrofocusing.

Dr. Muriel Jansson, Image Analytics, talked about a new laser-based densitometer that is designed to scan wet two-dimensional gels and autoradiograms with high resolution. The instrument accomplishes digitization of the protein images using a 12 bit analog-todigital converter to provide 4,096 gray levels. This device is able to scan a gel and provide all of the integrated data in about 30 minutes. The device automatically finds the spots of protein on a given gel, gives a printout of spot number, the coordinates, and the integrated total volume of each spot. It also produces correlations and matching between spots in different gels. It has a wide absorbance range that is linear up to 3 absorbance units and shows $0.1 \%$ scattering.

Dr. Catherine Phillips, DuPont, spoke on detection and quantification of radioactive proteins in gels using new autoradiographic methods. Using a commonly available video-based system, she found that there was a curvilinear relationship between spot volume and concentration of proteins stained with Coomassie Blue stain. The laser-based system, previously described by Dr. Jansson, provided a linear response between the amount of stain and the amount of protein in the stained gels. Nevertheless, fluorometric and autoradiographic methods for protein detection still proved to be more sensitive. The accurate quantification of protein and peptide "spots" in a two-dimensional gel are much more problematic. This is due to the nonlinear relationship of the photographic density generated by

... the need for particle standards will become increasingly

\section{important.}

radioactivity of labelled proteins. Dr. Phillips described software to transform image data into uniformly-spaced gray levels correlated with the amount of radioactivity present in the protein. In addition, she described optimization of the image recording system by use of an astrophotographic process termed "hypersensitization" of the photographic emulsion.

Dr. Phillips concluded her talk by discussing the evidences that calibration and step tablets are essential in standardization of the electrophoretic quantification process.

Dr. Robert Snyder, National Aeronautic and Space Administration (NASA), presented information on particle standards for continuous flow electrophoresis. This technique of free-flow electrophoresis is best applied to the separation of cells and large molecules. The major difficulties in achieving reproducibility in flow electrophoresis are ascribed to buoyancy and convection perturbations. Disturbing forces can occur when temperature gradients of $0.1^{\circ} \mathrm{C}$ exist.

Dr. Snyder summarized electrophoresis experiments performed in space over the history of the NASA programs. The early experiments showed the sensitivity of the electrophoresis separations to fluid viscosity changes due to electroosmosis. Cells were separated on a variety of space missions as parameters for electrophoretic separations in space were determined.

Polystyrene latex beads were separated by continuous flow electrophoresis into homogeneous fractions. Dr. Snyder suggested that as this work progresses, the need for particle standards will become increasingly important.

The meeting concluded with a roundtable discussion on directions for standardization, with Dr. R. C. Allen, Dr. Douglas Gersten, Dr. Marvin Lasky, and Dr.

The complexities and usefulness of standardization were realized ...

David Sammons as participants. Presentations focused on the current status of protein molecular weight markers and the potential for new markers for isoelectric standards as well as molecular weight markers.

In summary, the workshop was useful in bringing to light several approaches to standardization in electrophoresis. Various manufacturers of electrophoretic equipment and chemicals are interested in providing materials that will meet the needs of researchers who are extending the capabilities of electrophoresis into the analysis of smaller amounts of sample with better precision.

The complexities and usefulness of standardization were realized by the participants. Several attendees expressed willingness to be involved in a trial to test interlaboratory comparability in electrofocusing. The initial study is scheduled to begin this year as an interlaboratory round-robin to test the effectiveness of rehydratable gels for obtaining consistent patterns with standard protein preparation. NBS will serve as the coordinator and provide data analysis to determine the potential of such studies. Dr. Allen will prepare the rehydratable gels and a standard protein will be supplied by NBS.

Prepared by Dennis J. Reeder of the Organic Analytical Research Division, National Bureau of Standards, Gaithersburg, MD 20899. 